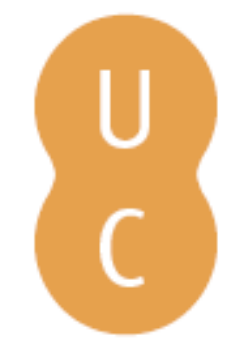

\title{
nombalina
}

\section{Os SIG na avaliação da vulnerabilidade estrutural e social associada a tsunamis: aplicação aos concelhos de Vila do Bispo e Figueira da Foz}

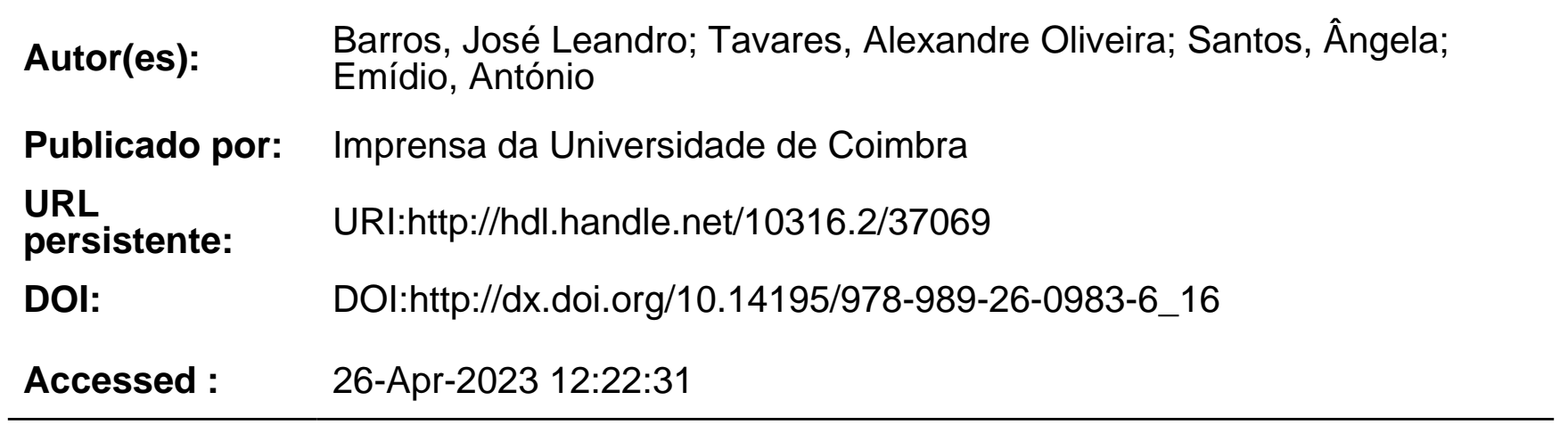

A navegação consulta e descarregamento dos títulos inseridos nas Bibliotecas Digitais UC Digitalis, UC Pombalina e UC Impactum, pressupõem a aceitação plena e sem reservas dos Termos e Condições de Uso destas Bibliotecas Digitais, disponíveis em https://digitalis.uc.pt/pt-pt/termos.

Conforme exposto nos referidos Termos e Condições de Uso, o descarregamento de títulos de acesso restrito requer uma licença válida de autorização devendo o utilizador aceder ao(s) documento(s) a partir de um endereço de IP da instituição detentora da supramencionada licença.

Ao utilizador é apenas permitido o descarregamento para uso pessoal, pelo que o emprego do(s) título(s) descarregado(s) para outro fim, designadamente comercial, carece de autorização do respetivo autor ou editor da obra.

Na medida em que todas as obras da UC Digitalis se encontram protegidas pelo Código do Direito de Autor e Direitos Conexos e demais legislação aplicável, toda a cópia, parcial ou total, deste documento, nos casos em que é legalmente admitida, deverá conter ou fazer-se acompanhar por este aviso.

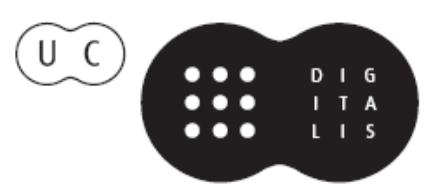




\section{$\forall$ \\ TAS DAS I JORNADAS LUSÓFONAS DE CIÊNCIAS E TECNOLOGIAS DE INFORMAÇÃO GEOGRÁFICA}

Editores

José Gomes dos Santos

Cidália Fonte

Rui Ferreira de Figueiredo

Alberto Cardoso

Gil Gonçalves

José Paulo Almeida

Sara Baptista 


\section{A RTIGO 16}

\section{OS SIG NA AVALIAÇÃO DA VULNERABILIDADE \\ ESTRUTURAL E SOCIAL ASSOCIADA A TSUNAMIS: APLICAÇÃo \\ AOS CONCElHos De Vila do Bispo E Figueira da Foz}

BARROS, José Leandro'; TAVARES, Alexandre Oliveira²;

SANTOS, Ângela ${ }^{3}$ \& EMÍDIO António ${ }^{3}$

\footnotetext{
${ }^{1}$ Centro de Estudos Sociais da Universidade de Coimbra (Portugal); Colégio de S. Jerónimo Largo D. Dinis Apartado 3087 3000-995, Coimbra (Portugal); email: leandrobarros@ces.uc.pt

2 Centro de Estudos Sociais e Departamento de Ciências da Terra, Universidade de Coimbra (Portugal); Largo Marquês de Pombal, 3000-272, Coimbra (Portugal); email: atavares@ci.uc.pt

3 Instituto de Geografia e Ordenamento do Território, Universidade de Lisboa, (Portugal) Edifício da Faculdade de Letras, Alameda da Universidade, 1600-214,Lisboa, (Portugal) email: angela.santos@campus. ul.pt; a.emidio@campus.ul.pt
}

\section{RESUMO}

Diversas metodologias têm sido desenvolvidas com o objectivo de avaliar a vulnerabilidade dos edifícios face a tsunamis. O presente trabalho apresenta a aplicação de uma análise integrada multidimensional de avaliação da vulnerabilidade a tsunamis para o concelho da Figueira da Foz e de Vila do Bispo, Portugal. São calculados índices de vulnerabilidade estrutural e social para a área potencialmente inundada utilizando como referência a modelação numérica da inundação do Tsunami de Lisboa de 1755.

\section{PALAVRAS-CHAVE}

Tsunami de Lisboa de 1755, Vulnerabilidade, SIG, Figueira da Foz, Vila do Bispo 


\title{
GIS IN THE ASSESSMENT OF STRUCTURAL AND SOCIAL VULNERABILITY ASSOCIATED WITH TSUNAMIS: \\ APPLICATION TO THE MUNICIPALITIES OF \\ VILA DO BISPO AND FIGUEIRA DA FOZ
}

\begin{abstract}
Several methodologies have been developed with the aim of assessing the vulnerability of buildings to tsunamis. This paper presents the application of a multidimensional integrated analysis for the vulnerability assessment to tsunamis for the municipality of Figueira da Foz and Vila do Bispo, Portugal. The structural and social vulnerability indices are calculated to potentially inundated areas using as reference the inundation numerical modeling of the 1755 Lisbon Tsunami.
\end{abstract}

\section{KEYWORDS}

1755 Lisbon Tsunami, Vulnerability, GIS, Figueira da Foz, Vila do Bispo

\section{INTRODUÇÃO}

Os registos históricos permitem identificar um total de 17 tsunamis ocorridos ao longo da costa portuguesa desde 60 a.C. até à actualidade (BAPTISTA e MIRANDA, 2009) sendo que 14 estão relacionados com a ocorrência de sismos. Destaca-se o tsunami ocorrido no dia 1 de Novembro de 1755 que resultou no maior desastre natural ocorrido em Portugal tendo provocado um elevado número de vítimas e estragos significativos em várias zonas do território continental, com maior incidência na cidade de Lisboa (SANTOS, 2008). O último tsunami que afectou a costa portuguesa ocorreu em 1969 e o intervalo de tempo de 45 anos que o separa da actualidade faz com que várias gerações não tenham convivido com a manifestação de eventos deste tipo. Esta ausência faz com que haja um falso sentimento de segurança, o que condiciona a percepção da população em relação ao risco de tsunami. Segundo Tavares et al. (2011) em Portugal os valores relacionados com a percepção face a riscos naturais e tecnológicos são genericamente baixos, sendo esta tendência mais clara nas zonas mais próximas dos locais de residência. O mesmo estudo conclui que quanto maior é a proximidade à zona de residência 
menor é a intensidade percebida dos processos perigosos, influenciada pela noção de segurança e controlo dos acontecimentos. O estudo levado a cabo por Mendes e Freiria (2012) do observatório do Risco OSIRIS demonstra que num conjunto de 28 riscos, os tsunamis surgem em último lugar numa lista que identifica a importância dos riscos percepcionados pelos portugueses. Por outro lado o estudo realça ainda que $95 \%$ dos inquiridos não adoptam qualquer medida de prevenção face a tsunamis, concluindo que a percepção do risco de tsunami em Portugal é muito baixa, mesmo para as populações que habitam na zona costeira ou em estuários.

Vários estudos realçam a importância da avaliação da vulnerabilidade associada a tsunamis, com especial incidência para a vulnerabilidade estrutural dos edifícios potencialmente afectados (PAPATHOMA et al., 2003; PAPATHOMA E DOMINEY-HOWES, 2003; DOMINEY-HOWES P PAPATHOMA, 2007; DALL'OSSO et al., 2009; KAPLAN et al., 2009; DALL'OSSO et al., 2010; ANPC, 2010; OMIRA et al., 2010; LEONE et al., 2011; RIBEIRO et al., 2011; ISMAIL et al., 2012; BARROS et al., 2013; EMÍDIO et al., 2013), sendo adequadas para a análise da vulnerabilidade na escala local. No entanto, independentemente da escala, a vulnerabilidade apresenta uma variabilidade ao longo do território, estando dependente das características físicas e socio-económicas. Estes dois pilares de análise não estão presentes na maioria das metodologias de análise de vulnerabilidade face a tsunamis, o que constitui uma das suas principais limitações (DALL'OSSO et al. 2010) originando uma análise de vulnerabilidade "incompleta". Assim sendo, para além da vertente estrutural é necessário incluir outras componentes nomeadamente a social na avaliação da vulnerabilidade face a tsunamis. Por outro lado, vários autores sublinham também a relevância da análise de vulnerabilidade social e da identificação de grupos de risco como chave para o processo de avaliação do risco (CUTTER et al. 2003; FEKETE et al. 2009; MENDES, 2009; MENDES et al. 2011; BIRKMANN et al. 2011).

O presente estudo é inovador no sentido em que dá a conhecer uma análise integrada da vulnerabilidade, pelo que se distingue das demais metodologias pelo facto de ser uma abordagem multidimensional. Esta 
abordagem agrega na sua composição a componente estrutural e social da vulnerabilidade, tendo por base os estudos realizados por Cutter et al. (2003), Schmidtlein et al. (2008) e Mendes (2009) no que concerne à componente social, e Dall'Osso et al. (2009) para a componente estrutural. Inclui-se assim a vulnerabilidade social como elemento diferenciador em relação a outras abordagens, assim como se refinam os processos de análise ao introduzir novos parâmetros na avaliação da vulnerabilidade estrutural. A definição das áreas potencialmente afectadas é realizada com recurso à modelação numérica de inundação por tsunami, tendo como referência o tsunami de 1 de Novembro de 1755 (SANTOS, 2008; SANTOS et al. 2009; SANTOS et al. 2011; SANTOS et al. 2012; SANTOS \& KOSHIMURA, 2013a).

\section{TEMA E ÁREAS DE ESTUDO}

O concelho da Figueira da Foz (Figura 1) está localizado na zona centro litoral de Portugal Continental e apresenta uma área de $379 \mathrm{~km}^{2}$. Segundo os Censos 2011 (INE, 2011) o concelho regista uma população de 62125 habitantes, repartida de forma desigual pelas 18 freguesias, com uma densidade populacional de 163.3 (hab/ $\mathrm{km}^{2}$ ). No espaço concelhio destaca-se a Figueira da Foz como uma cidade de média dimensão no panorama nacional com um total de 28338 habitantes. A escolha da Figueira da Foz justifica-se pelo facto da existência de relatos históricos relacionados com o tsunami de 1755 (SANTOS, 2008; SANTOS et al. 2009; SANTOS et al. 2011; SANTOS et al. 2012; SANTOS \& KOSHIMURA,

2013b), a elevada flutuação sazonal da população, a existência de um importante porto e zona industrial junto à costa.

O concelho de Vila do Bispo, localiza-se na zona mais a sudoeste do território continental (Figura 1), inserindo-se na região denominada de Barlavento Algarvio e com uma área de

$179 \mathrm{~km}^{2}$. De acordo com os Censos 2011 (INE, 2011) o concelho apresenta uma população de 5381 habitantes, abrangendo o município um total de 5 freguesias apresentando uma baixa densidade populacional $\left(29,2 \mathrm{hab} / \mathrm{km}^{2}\right)$. No que respeita a aglomerados populacionais destaca-se 
Vila do Bispo que é a sede de concelho e se localiza na zona centro do mesmo. Junto à costa destacam-se Sagres, Salema e Burgau como locais de maior concentração populacional. A escolha de Vila do Bispo justifica-se pela existência de relatos históricos associados ao tsunami de 1755 (SANTOS, 2008; SANTOS et al. 2009; SANTOS et al. 2011; SANTOS \& KOSHIMURA, 2013b). Outra razão prende-se com o facto de um tsunami com uma origem semelhante ao verificado em 1755 demorar menos de 20 minutos atingir o concelho. Por último, a existência de aglomerados populacionais junto à costa (Sagres, Burgau e Salema) e a sua considerável flutuação populacional sazonal justificam o estudo.

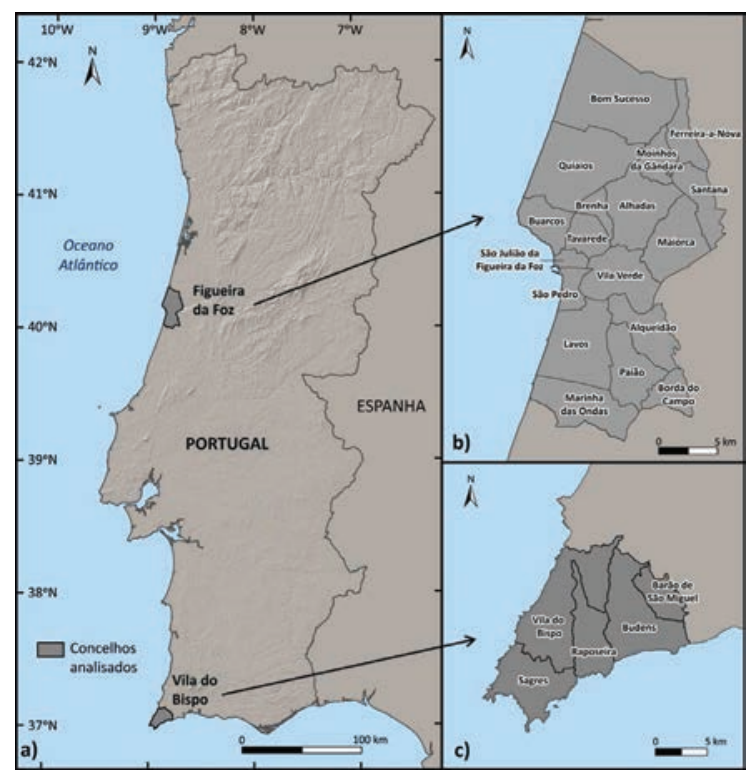

Figura 1 - Enquadramento área de estudo

\section{METODOLOGIA}

A análise integrada presente no artigo caracteriza-se pela sua componente multidimensional que agrega componentes estrutural e social da vulnerabilidade. Para a execução da metodologia foi criada uma matriz de análise que agrega todos os parâmetros que compõem a componente estrutural da vulnerabilidade. A matriz apresenta um conjunto de atributos intrínsecos a cada parâmetro e atribui a cada um, valores que variam entre 1 e -1 , em que os valores positivos representam um aumento da 
vulnerabilidade e os valores negativos um decréscimo da mesma. A matriz serviu de base a trabalho de campo realizado com recurso a tecnologia SIG (ArcGis 10.2 (ESRI®) e Android® através da utilização de um tablet para a recolha de informação no terreno. O trabalho foi complementado com recurso a imagens de satélite, nomeadamente o serviço World Imagery disponibilizado pela ESRI®.

No que concerne à análise da componente estrutural foram analisados os edifícios potencialmente afectados pela modelação numérica de inundação face a tsunamis (SANTOS, 2008; SANTOS et al. 2012). Os edifícios resultaram da análise de imagens de satélite (ESRI, 2014) e de trabalho de campo que permitiram a representação vectorial e adição de atributos intrínsecos à tipologia, forma, ocupação e funcionalidade. É de salientar a dificuldade de obter uma "linha de costa" coerente face à erosão e deposição das areias da costa portuguesa (SANTOS et al. 2012; INAG, 2009). A linha de costa considerada resultou da actualização da cartografia do IGeoE - Instituto Geográfico do Exército, nomeadamente das cartas militares de Portugal, serie M888 (2000, 2001, 2002) com dados de levantamento topográfico a partir de GPS. Para a avaliação do edificado foram seleccionados 13 parâmetros que caracterizam os edifícios na componente estrutural, arquitectónica e ocupacional (Tabela 1). Nesta componente para além da introdução de parâmetros presentes em Dall'Osso et al. (2009) e Ismail et al. (2012) propõe-se neste estudo um conjunto de novos parâmetros (Tabela 1), de onde se destacam os relacionados com a ocupação em cada edifício (ano de construção; forma de ocupação; número de unidades de utilização; número médio diário de pessoas presentes ou visitantes por unidade de utilização e flutuação da ocupação), permitindo complementar a análise dos edifícios nomeadamente através da sua distinção funcional, sazonal e ocupacional. Nem todos os parâmetros contribuem da mesma forma para o cálculo da vulnerabilidade estrutural, como tal recorreu-se ao software de análise multicritério M-Macbeth®, que através da comparação directa entre os diferentes parâmetros permitiu atribuição de ponderações consoante a importância de cada um (BANA e COSTA, 2004; BANA e COSTA \& CHAGAS, 2004). 
Tabela 1 - Parâmetros de avaliação da vulnerabilidade estrutural, propostos neste estudo

\begin{tabular}{|c|c|}
\hline & 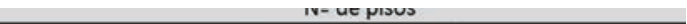 \\
\hline & Material de construção \\
\hline & Ano construção \\
\hline & Estado conservação \\
\hline & Hidrodinâmica do $\mathrm{r} / \mathrm{c}$ \\
\hline ros de avaliação da & Existência pisos subterrâneos \\
\hline bilidade estrutural & Tipo de fundações \\
\hline & Forma ocupação \\
\hline & № unidades de utilização \\
\hline & № médio de pessoas presentes ou visitantes por unidades de utilizas \\
\hline & $\begin{array}{l}\text { Flutuação ocupação } \\
\end{array}$ \\
\hline & Forma edificado em planta \\
\hline
\end{tabular}

Por último foi avaliada a vulnerabilidade social tendo por base a metodologia preconizada por Cutter et al. (2003), Schmidtlein et al. (2008) e Mendes (2009) com recurso à análise factorial realizada em SPSS. Neste estudo propuseram-se um total inicial de 172 variáveis (INE, 2013; PORDATA, 2013; CARTA SOCIAL, 2013) de origem social, económica, demográfica e relacionadas com o edificado da área de estudo. Foi considerado nesta avaliação os dados referentes à freguesia, dado que esta constitui o nível mínimo de desagregação para múltiplas variáveis (ex. económicas, apoio social, recursos de protecção civil, entre outros).

Para se aumentar a robustez do método utilizaram-se ainda 88 freguesias de 15 concelhos da orla costeira. Os cálculos foram efectuados com a utilização de SPSS. Após a eliminação dos dados redundantes a análise factorial foi executada até serem atingidos um conjunto de parâmetros que validassem a amostra e que no presente estudo se traduziu num KMO de 0,717, comunalidades superiores a 0,6 e uma taxa de variância de $78 \%$. Estes resultados conduziram a um total de 47 variáveis, como é apresentado na Tabela 2. Após concluída a análise factorial foi calculada a vulnerabilidade social para cada freguesia com base na classificação de Cutter et al. (2003), onde os autores classificam a vulnerabilidade entre muito baixa e muito elevada. 
Tabela 2 - Variáveis utilizadas no cálculo da vulnerabilidade social, propostas neste estudo

\begin{tabular}{|c|c|}
\hline Agricultura & $\begin{array}{l}\text { Proporçăo produtores agricolas singulares } c 0 m 65 \text { e mais anos de idade }(\%) \\
\text { Proporçăo de produtores agricolas singulares }(\%) \text { sem nenhum nivel de ensino } \\
\text { Proporçăo de produtores agricolas singulares }(\%) \text { com ensino básico }\end{array}$ \\
\hline $\begin{array}{l}\text { Edifícios } \\
\text { e } \\
\text { Alojamentos }\end{array}$ & 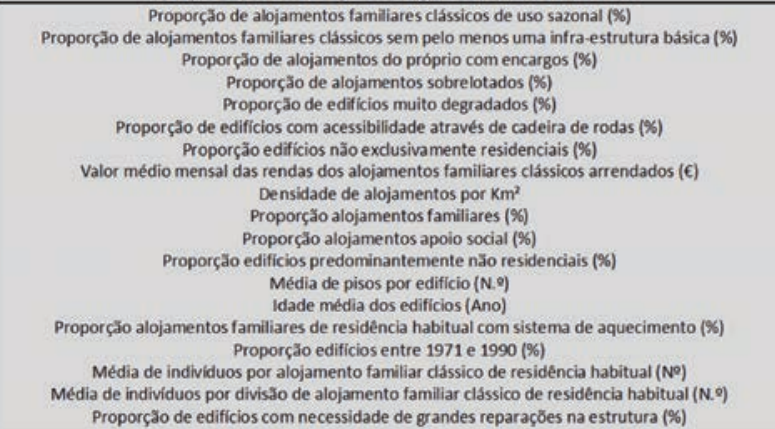 \\
\hline Economia & 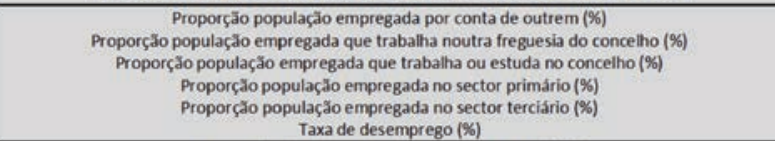 \\
\hline Populaçăo & 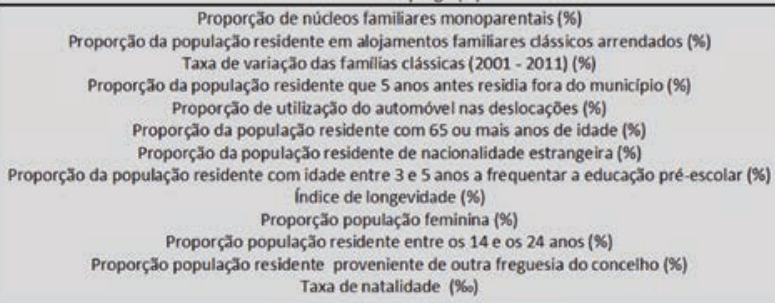 \\
\hline Serviços & $\begin{array}{l}\text { Habitantes por centro de saúde e extensăo }\left(\mathrm{n}^{\circ}\right) \\
\text { Bombeiros por } 1000 \text { habitantes } \\
\text { Estada media nos estabelecimentos hoteleiros (N.9) }\end{array}$ \\
\hline Apoios socials & $\begin{array}{l}\text { Populaçăo adulta institucionalizada por } 100 \text { habitantes } \\
\text { Infra-estruturas de infância e juventude por } 100 \text { habitantes } \\
\text { Infra-estruturas para populaçăo adulta por } 100 \text { habitantes }\end{array}$ \\
\hline
\end{tabular}

\section{RESULTADOS}

\subsection{Vulnerabilidade estrutural}

A análise da vulnerabilidade estrutural permitiu a identificação de um total de 244 edifícios potencialmente afectados pela ocorrência de um tsunami com características semelhantes ao de 1755. No concelho da Figueira da Foz identificam-se 144 edifícios com destaque para as zonas do Cabedelo, porto da Figueira da Foz, Cova Gala e Leirosa (Figura 2) 
como as áreas mais afectadas. No concelho de Vila do Bispo foram identificados um total de 100 edifícios, com destaque para as zonas de Sagres, Salema e Burgau (Figura 3) como as regiões mais afectadas.

No concelho da Figueira da Foz a vulnerabilidade varia entre baixa e elevada, sendo que $85 \%$ dos edifícios localizam-se na classe média, $14 \%$ na classe elevada e $1 \%$ na classe baixa. Tipologicamente $51 \%$ dos edifícios identificados são exclusivamente residenciais, 13\% anexos, 10\% comerciais, 10\% armazéns/estaleiros e 5\% edifícios agrícolas. A margem esquerda do Mondego concentra a grande maioria dos edifícios potencialmente afectados (92\%) onde se destaca a área do Cabedelo com um total de 22 edifícios, o porto com 10, a Cova Gala com 15 e a Leirosa com 85 edifícios.

Em Vila do Bispo (Figura 3) a vulnerabilidade estrutural varia entre muito baixa e elevada, sendo que $82 \%$ dos edifícios estão localizados na classe média, $12 \%$ na classe baixa, $5 \%$ na classe elevada e $1 \%$ na classe muito baixa, como mostra a Figura 2. Em termos da tipologia do edificado destaque para o facto de $47 \%$ serem exclusivamente residenciais, $20 \%$ exclusivamente comerciais e $8 \%$ são residenciais/comerciais. $\mathrm{Na}$ área do concelho destacam-se a praia da Salema com um total de 41 edifícios potencialmente afectados, a praia do Burgau com 25 edifícios e em Sagres, nomeadamente na zona da Baleeira e do porto onde são identificados um total de 10 edifícios. 


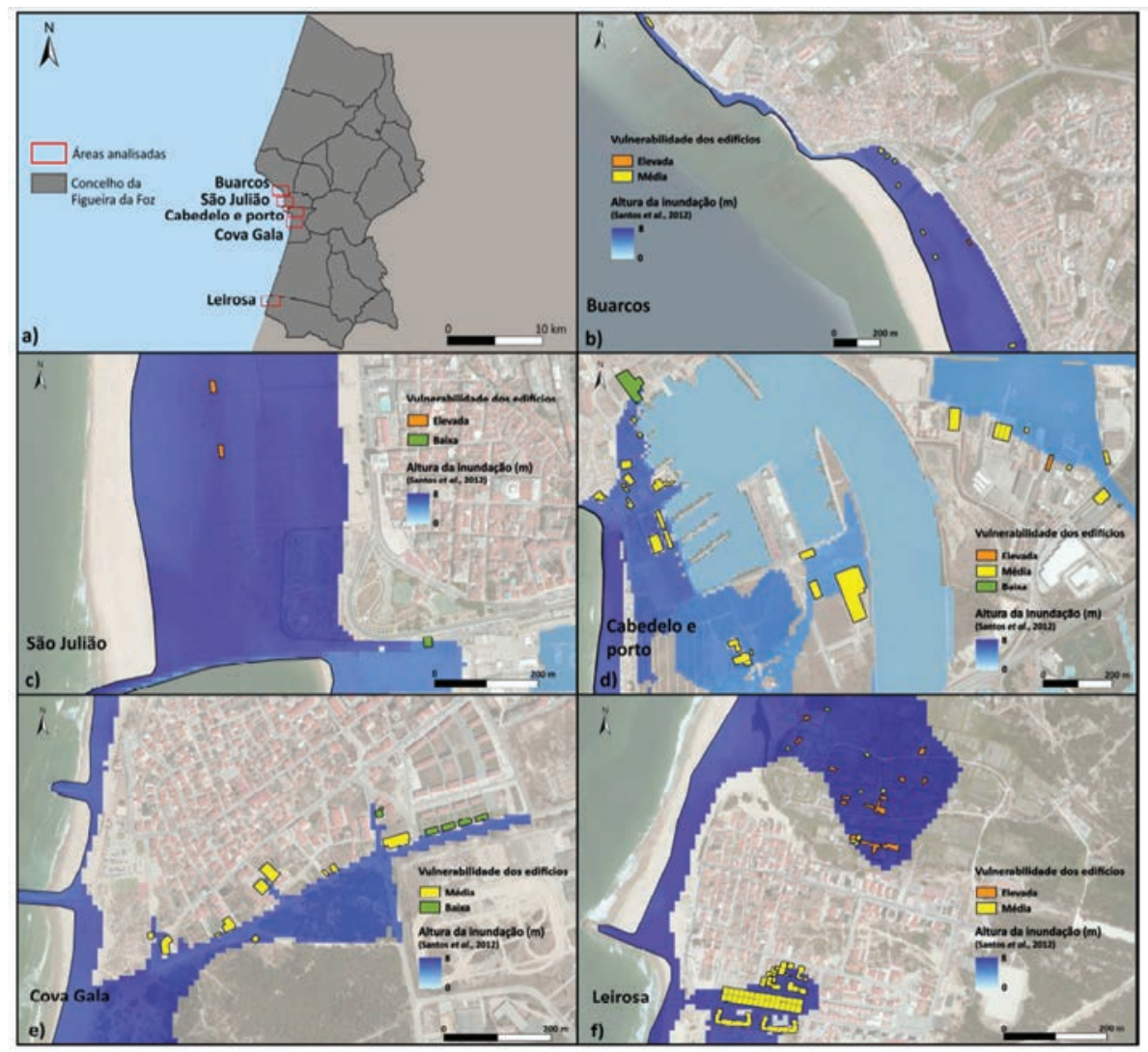

Figura 2 - Principais áreas potencialmente afectadas no concelho da Figueira da Foz

\subsection{Vulnerabilidade social}

No que respeita à Figueira da Foz a Tabela 3 permite constatar que a vulnerabilidade social do concelho varia entre baixa e muito elevada, sendo que uma freguesia apresenta vulnerabilidade baixa, 11 com vulnerabilidade média, 3 com vulnerabilidade elevada e 3 com vulnerabilidade muito elevada. No que concerne às áreas potencialmente mais afectadas o Cabedelo, o porto industrial da Figueira e a Cova Gala localizam-se na freguesia de São Pedro e apresentam uma vulnerabilidade social muito elevada. A Leirosa, localizada na zona sul do concelho na freguesia de Marinha das Ondas apresenta uma vulnerabilidade baixa. 


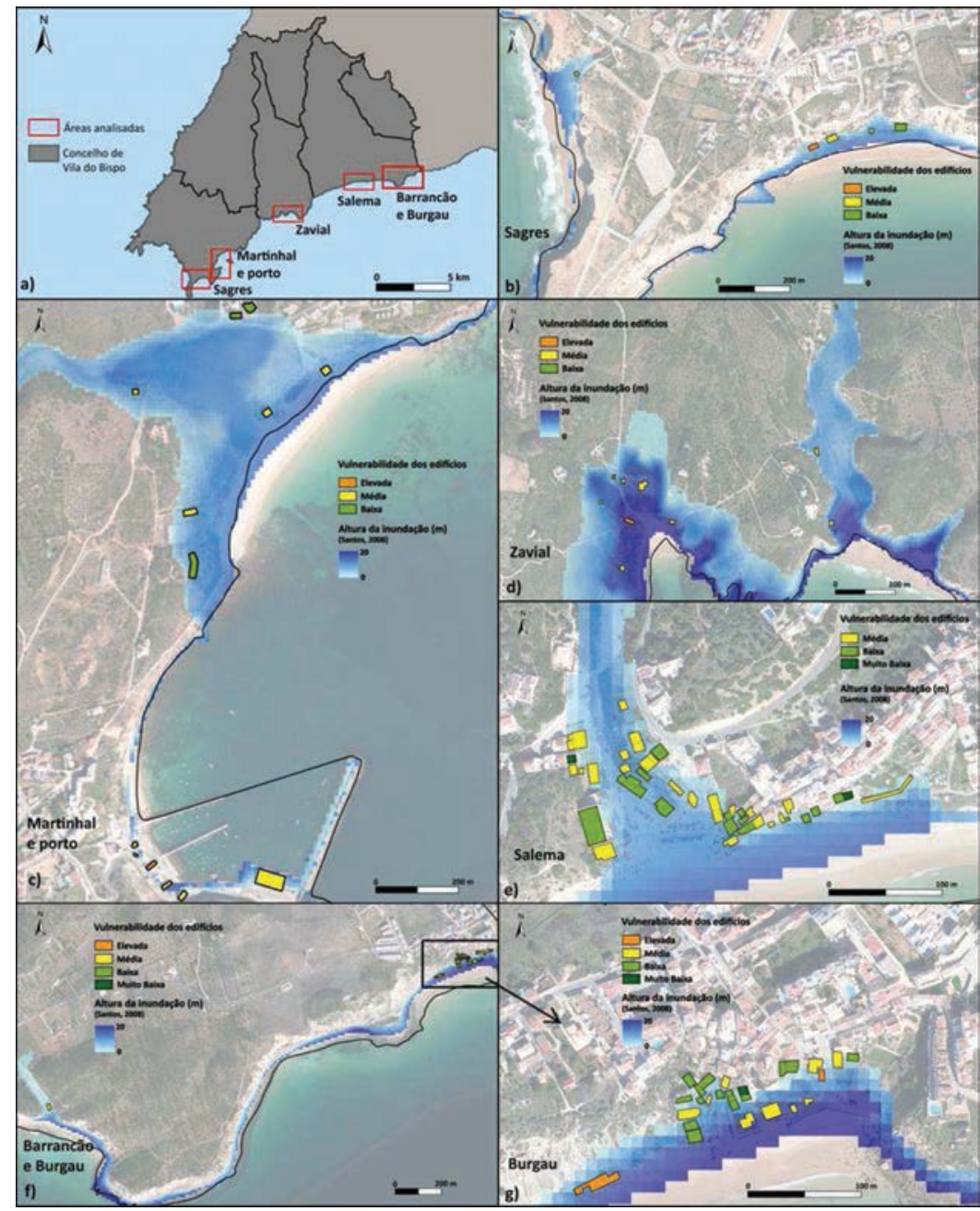

Figura 3 - Principais áreas potencialmente afectadas no concelho de Vila do Bispo

O concelho de Vila do Bispo patente na Tabela 3 mostra que a vulnerabilidade social do concelho varia entre muito baixa e muito elevada. Das cinco freguesias que constituem o concelho uma freguesia apresenta vulnerabilidade muito baixa, duas com vulnerabilidade média, uma com elevada e uma com muito elevada. No que respeita às áreas potencialmente mais afectadas verifica-se que Sagres, inserida na freguesia com o mesmo nome, apresenta uma vulnerabilidade média. Na freguesia de 
Budens localiza-se a Salema e o Burgau que apresenta uma vulnerabilidade social elevada.

Tabela 3 - Vulnerabilidade social no concelho da Figueira da Foz e de Vila do Bispo

\begin{tabular}{|c|c|c|c|c|}
\hline Concelhos & Freguesias & $\begin{array}{c}\text { Valor } \\
\text { Vulnerabilidade }\end{array}$ & $\begin{array}{c}\text { Grau } \\
\text { vulnerabilidade }\end{array}$ & Áreas analisadas \\
\hline \multirow{18}{*}{ Figueira da Foz } & Brenha & 0.73 & \multirow{3}{*}{ Muito Elevado } & \multirow{3}{*}{$\begin{array}{c}\text { Cabedelo e } \\
\text { porto } \\
\text { Cova Gala }\end{array}$} \\
\hline & Vila Verde & 0.70 & & \\
\hline & São Pedro & 0.70 & & \\
\hline & Santana & 0.64 & \multirow{3}{*}{ Elevado } & \\
\hline & Alhadas & 0.61 & & \\
\hline & Quiaios & 0.59 & & \\
\hline & Buarcos & 0.54 & \multirow{11}{*}{ Médio } & \multirow{11}{*}{ São Julião } \\
\hline & Borda do Campo & 0.53 & & \\
\hline & Lavos & 0.52 & & \\
\hline & Tavarede & 0.50 & & \\
\hline & Maiorca & 0.50 & & \\
\hline & Bom Sucesso & 0.48 & & \\
\hline & $\begin{array}{c}\text { Moinhos da } \\
\text { Gândara }\end{array}$ & 0.46 & & \\
\hline & São Julião & 0.46 & & \\
\hline & Paião & 0.46 & & \\
\hline & Alqueidão & 0.44 & & \\
\hline & Ferreira-a-Nova & 0.42 & & \\
\hline & $\begin{array}{l}\text { Marinha das } \\
\text { Ondas }\end{array}$ & 0.39 & Baixo & Leirosa \\
\hline \multirow{7}{*}{ Vila do Bispo } & $\begin{array}{c}\begin{array}{c}\text { Barão de São } \\
\text { Miguel }\end{array} \\
\end{array}$ & 0.77 & Muito Elevado & \\
\hline & \multirow{3}{*}{ Budens } & \multirow{3}{*}{0.65} & \multirow{3}{*}{ Elevado } & Salema \\
\hline & & & & Barrancão \\
\hline & & & & Burgau \\
\hline & Sagres & 0.53 & \multirow[b]{2}{*}{ Médio } & Sagres \\
\hline & Vila do Bispo & 0.47 & & $\begin{array}{l}\text { Martinhal e } \\
\text { porto }\end{array}$ \\
\hline & Raposeira & 0.30 & Muito Baixo & Zavial \\
\hline
\end{tabular}

\section{CONCLUSÕES}

A análise integrada multidimensional utilizada no presente trabalho apresenta-se como uma boa solução para a análise do edificado potencialmente afectado por tsunami. A introdução de novos parâmetros (ano de construção; forma de ocupação; número de unidades de utilização; 
número médio diário de pessoas presentes ou visitantes por unidade de utilização e flutuação da ocupação), em relação a metodologias precedentes permitiu completar e aumentar a robustez da análise, nomeadamente através da sua distinção funcional, sazonal e ocupacional. Por outro lado o recurso aos SIG e ao sistema operativo Android®, permitiu simplificar a recolha dos dados no terreno bem como facilitar a avaliação da vulnerabilidade ao longo do tempo e do espaço. Por último a realização da análise factorial com recurso ao SPSS ${ }^{\circledR}$ permitiu robustecer o modelo através da selecção de variáveis utilizadas no cálculo da vulnerabilidade social.

Ao longo do estudo foram identificados e avaliados 244 edificios de onde se conclui que $83 \%$ apresentam uma vulnerabilidade média, 11\% vulnerabilidade elevada, 5\% vulnerabilidade baixa e 1\% vulnerabilidade muito baixa.

Como limitações metodológicas apontam-se: o acesso a uma linha de costa actualizada e suportando o modelo de inundação; a caracterização de edificado a partir de observações de campo não validadas por bases de dados de cadastro municipal; a limitação na desagregação de múltiplas variáveis à escala do lugar; a necessidade de introdução de outras variáveis estruturais e sociais de melhor resolução local. O cálculo da vulnerabilidade social permite complementar a componente estrutural da metodologia permitindo trazer para a análise a avaliação das diferentes variáveis territoriais. Estes resultados permitem concluir que existem diferenças entre os concelhos em análise: na Figueira da Foz a vulnerablidade social varia entre baixa e muito elevada, com a maioria das freguesias apresentar uma vulnerabilidade média, enquanto que em Vila do Bispo a variação é entre muito baixa e muito elevada. Os resultados apresentados neste estudo devem servir como ponto de partida para as autoridades competentes, para a reflexão e adopção de medidas de mitigação do risco associado a tsunamis.

\section{AgRAdecimentos}

Este estudo foi suportado pelo Projeto Tsurima - Gestão do risco de tsunamis para o ordenamento do território e a protecção civil, PTDC/CS- 
GEO/118992/2010), financiado pela Fundação para a Ciência e Tecnologia (FCT), Portugal.

\section{BIBLIOGRAFIA}

ANPC (2010) - "Estudo do Risco Sísmico e de Tsunamis do Algarve (ERSTA)". Autoridade de Protecção Civil. 85 pp. Disponível online no endereço url: http://www.prociv.pt/Pages/detalhe4.aspx?IDitem=58 (acedido em 1 Fevereiro, 2014)

BANA e COSTA, Carlos António \& CHARGAS, Manuel (2004) - "An example of how to use MACBETH to build a quantitative value model based on qualitative value judgements”. Eur. J. Oper. Res., 153, 323-331, Disponível online no endereço url: http://www.emeraldinsight.com/bibliographic_databases. htm?id=1313655\&show=abstract (acedido em 1 Fevereiro, 2014)

BANA e COSTA, Carlos António (2004) - "Multicriteria Evaluation of Flood Control Measures: The case of Ribeira do Livramento". Water Resour. Manag., 18, 263-283, 2004. Disponível online no endereço url: http://link.springer. com/article/10.1023\%2FB\%3AWARM.0000043163.19531.6a (acedido em 3 Fevereiro, 2014)

BAPTISTA, Maria Ana \& MIRANDA, Jorge (2009) - "Revision of the Portuguese catalog of tsunamis". Nat. Hazards Earth Syst. Sci., 9, 25-42, doi:10.5194/ nhess-9-25-2009. Disponível online no endereço url: http://www.nat-hazards-earth-syst- sci.net/9/issue1.html (acedido em 3 Fevereiro, 2014).

BARROS, José Leandro et al. (2013) - "Metodologias de avaliação da vulnerabilidade ao risco de tsunami: aplicação ao sector costeiro Cova Gala - Leirosa; Figueira da Foz". Pinto Correia, T.; Henriques, V.; Julião, R.P. (Eds.), IX Congresso da Geografia Portuguesa - Geografia: Espaço, Natureza, Sociedade e Ciência, p. 839-845, Evora, Portugal, 28-30 Novembro, 2013. ISBN:978972-99436-6-9. Disponível online no endereço url: https://sites.google. com/a/campus.ul.pt/tsurima/publications (acedido em 1 Fevereiro, 2014)

BIRKMANN, Jörn et al. (2011) - "O risco não percepcionado para as zonas costeiras da Europa: Os tsunamis e a vulnerabilidade de Cádis, Espanha”. Revista Crítica de Ciências Sociais, Vol. 93, 129-165.

CARTA SOCIAL (2013) - http://www.cartasocial.pt, Gabinete de Estratégio e Planeamento, (acedido em 20 de Dezembro, 2013).

CUTTER, Susan et al. (2003) - "Social Vulnerability to Environmental Hazards". Social Science Quarterly, 84(2): 242-261. Disponível online no endereço url: http://onlinelibrary.wiley.com/doi/10.1111/1540-6237.8402002/abstract (acedido em 5 Fevereiro, 2014) 
DALL'OSSO, Filippo et al. (2009) - "Applying and validating the PTVA-3 Model at the Aeolian Islands, Italy: assessment of the vulnerability of buildings to tsunamis". Nat. Hazards Earth Syst. Sci., 10, 1547-1562, doi:10.5194/ nhess-10-1547-2010. Disponível online no endereço url: http://www.nat-hazards-earth-syst-sci.net/10/1547/2010/nhess- 10-1547-2010.html (acedido em 5 Fevereiro, 2014).

DALL'OSSO, Filippo et al. (2010) - "A novel approach (the CRATER method) for assessing tsunami vulnerability at the regional scale using ASTER imagery". Italian Journal of Remote Sensing 42(2): 55-74. Disponível online no endereço url: http://www.aitjournal.com/articleView.aspx?ID=164 (acedido em 5 Fevereiro, 2014).

DOMINEY-HOWES, Dale \& PAPATHOMA, Maria (2007) - "Validating a tsunami vulnerability assessment model (the PTVA model) using field data from the 2004 Indian Ocean tsunami”. Natural Hazards, 40, 113-136. Disponível online no endereço url: http://link.springer.com/article/10.1007/s11069006-0007-9\# (acedido em 1 Fevereiro, 2014).

EMÍDIO, António et al. (2013) - "Aplicação da metodologia PTVA-3 na avaliação da vulnerabilidade do centro urbano da cidade de Setúbal em caso de tsunami”. Pinto Correia, T.; Henriques, V.; Julião, R.P. (Eds.), IX Congresso da Geografia Portuguesa - Geografia: Espaço, Natureza, Sociedade e Ciência, p. 833-838, Evora, Portugal, 28-30 Novembro, 2013. ISBN:978-972-99436-6-

9. Disponível online no endereço url: https://sites.google.com/a/campus. ul.pt/tsurima/publications (acedido em 3 Fevereiro, 2014)

ESRI (2014) - World Imagery - Map Service Disponível online no endereço url: http://www.arcgis.com/home/item.html?id=10df2279f9684e4a9f6a7f08fe bac2a9 (acedido em 1 Fevereiro, 2014)

FEKETE, Alexander (2009) - Validation of a social vulnerability index in context to river- floods in Germany, Nat. Hazards Earth Syst. Sci., 9, 393-403, doi:10.5194/nhess-9-393- 2009. Disponível online no endereço url: http:// www.nat-hazards-earth-syst- sci.net/9/393/2009/nhess-9-393-2009.html (acedido em 3 Fevereiro, 2014).

IGEOE (2000) - Instituto Geográfico do Exército, CMP, Série M888, Edição 3

IGEOE (2001) - Instituto Geográfico do Exército, CMP, Série M888, Edição 3

IGEOE (2002) - Instituto Geográfico do Exército, CMP, Série M888, Edição 3

INAG (2009) - Instituto da Água. "Estratégia Nacional para a Gestão Integrada da Zona Costeira". Lisboa, 89 pp.

INE (2011) - Recenseamento Geral da População e da Habitação. Instituto Nacional de Estatística, IP - Portugal, Lisboa

INE (2013) - http://www.ine.pt, Instituto Nacional de Estatística, IP - Portugal, Lisboa, (acedido em 20 de Dezembro, 2013). 
ISMAIL, Hadibah et al. (2012) - "A 3-tier tsunami vulnerability assessment technique for the nort-west coast of Peninsular Malaysia”. Natural Hazards, 63, 549-573. Disponível online no endereço url: http://link.springer.com/ article/10.1007/s11069-012-0166-9\# (acedido em 1 Fevereiro, 2014).

KAPLAN, Marcus et al. (2009) - "Vulnerability assessment and protective effects of coastal vegetation during the 2004 Tsunami in Sri Lanka”. Nat. Hazards Earth Syst. Sci., 9, 1479- 1494, doi:10.5194/nhess-9-1479-2009. Disponível online no endereço url: http://www.nat-hazards-earth-syst-sci.net/9/1479/2009/ nhess-9-1479-2009.html (acedido em 5 Fevereiro, 2014).

LEONE, Frédéric et al. (2011) - "A spatial analysis of the December 26th, 2004 tsunami- induced damages: lessons learned for a better risk assessment integrating buildings vulnerability". Appl Geogr 31(1):363-375. Disponível online no endereço url: http://www.sciencedirect.com/science/article/pii/ S0143622810000834 (acedido em 5 Fevereiro, 2014)

MENDES, José Manuel (2009) - "Social Vulnerability Indexes as Planning Tools: Beyond the preparedness paradigm". Journal of Risk Research, 12(1), 43-58. Disponível online no endereço url: http:// www.tandfonline.com/doi/citedby/10.1080/13669870802447962\#tab Module (acedido em 1 Fevereiro, 2014).

MENDES José Manuel et al. (2011) - "A vulnerabilidade social aos perigos naturais e tecnológicos em Portugal”. Revista Crítica de Ciências Sociais, 93. 95-128 Disponível online no endereço url: http://www.ces.uc.pt/myces/UserFiles/ livros/1097_rccs-90-93-a- vulnerabilidade-social-aos-perigos-naturais-e-tecnologicos-em-portugal.pdf (acedido em 1 Fevereiro, 2014).

MENDES, José \& FREIRIA, Susana (2012) - "O risco de tsunami em Portugal: Percepções e Prácticas”. OSIRIS, Centro de Estudos Sociais da Universidade de Coimbra, Disponível online no endereço url: http://www.ces.uc.pt/ osiris/pages/pt/publicacoes/relatorios.php (acedido em 2 Fevereiro, 2014).

OMIRA, Rachid et al. (2010) - "Tsunami vulnerability assessment of CasablancaMorocco using numerical modelling and GIS tools". Natural Hazards, 54, 1, 75-95. Disponível online no endereço url: http://link.springer.com/article/10.1007/s11069-009-9454-4 (acedido em 2 Fevereiro, 2014)

PAPATHOMA, Maria, et al. (2003) - "Assessing tsunami vulnerability, an example from Herakleio". Crete, Nat. Hazards Earth Syst. Sci., 3, 377-389, doi:10.5194/ nhess-3-377- 2003. Disponível online no endereço url: http://www.nat-hazards-earth-syst- sci.net/3/377/2003/nhess-3-377-2003.html (acedido em 1 Fevereiro, 2014).

PAPATHOMA, Maria \& Dominey-Howes, Dale (2003) - "Tsunami vulnerability assessment and its implications for coastal hazard analysis and disaster management planning, Gulf of Corinth, Greece”. Nat. Hazards Earth Syst. Sci., 3, 733-747, doi:10.5194/nhess-3-733- 2003. Disponível online no endereço url: http://www.nat-hazards-earth-syst- sci.net/3/733/2003/nhess-3-733-2003. 
html (acedido em 1 Fevereiro, 2014).

PORDATA (2013) - http://www.pordata.pt, Fundação Francisco Manuel dos Santos, (acedido em 20 de Dezembro, 2013).

RIBEIRO, João et al. (2011) - "High resolution tsunami modelling for the evaluation of potential risk areas in Setúbal (Portugal)”. Nat. Hazards Earth Syst. Sci., 11, 2371-2380, doi:10.5194/nhess-11-2371-2011. Disponível online no endereço url: http://www.nat- hazards-earth-syst-sci.net/11/2371/2011/ nhess-11-2371-2011.html (acedido em 1 Fevereiro, 2014).

SANTOS Angela (2008) - Tsunami Hazard Assessment in Portugal by the Worst Case Scenario: The November 1st, 1755 Lisbon Tsunami, PhD Thesis, Tohoku University, 210 pp.

SANTOS Angela et al. (2009) - "The 1755 Lisbon Tsunami: Tsunami source determination and its validation". Jour. Dis. Res., 4:1, 41-52. Disponível online no endereço url: http://www.fujipress.jp/finder/xslt.php? $\operatorname{mode}=$ present\&inputfile=DSSTR000400010006.x ml (acedido em 4 Fevereiro, 2014)

SANTOS, Ângela et al. (2011) - "O tsunami de 1755 e a avaliação da perigosidade em Portugal continental”. VIII Congresso da Geografia Portuguesa, Repensar a Geografia para Novos Desafios, Comunicações, APG, Lisboa, 6 p., Outubro 26-29, Disponível online no endereço url: https://sites.google. com/a/campus.ul.pt/tsurima/publications (acedido em 4 Fevereiro, 2014)

SANTOS Ângela et al. (2012) - "Tsunami risk assessment at Figueira da Foz, Portugal”. 15 WCEE,Lisboa, Disponível online no endereço url: http:// riskam.ul.pt/images/pdf/paper_1931.pdf (acedido em 1 Fevereiro, 2014).

SANTOS, Lara, et al.(2012) - "Dealing with expertise and non expertise knowledge about coastal Risk". Vol. 83, pp 83-87 2nd World Conference on Educational Technology Researches - WCETR2012 Disponível online no endereço url: http://www.sciencedirect.com/science/article/pii/S187704281301046X (acedido em 1 Fevereiro, 2014).

SANTOS Angela \& Koshimura, Shunichi (2013a) - "Tsunami hazards at Setubal urban area considering the 1755 Lisbon Tsunami”. Pinto Correia, T.; Henriques, V.; Julião, R.P. (Eds.), IX Congresso da Geografia Portuguesa - Geografia: Espaço, Natureza, Sociedade e Ciência, p.930-935,Evora, Portugal, 28-30 Novembro,2013.ISBN:978-972-99436-6-9. Disponível online no endereço url: https://sites.google.com/a/campus.ul.pt/tsurima/publications (acedido em 4 Fevereiro, 2014)

SANTOS, Angela \& Koshimura, Shunichi (2013b) - "Estimating the tsunami parameters of the 1755 Lisbon Tsunami in Portugal by the interpretation of the historical accounts". Pinto Correia, T.; Henriques, V.; Julião, R.P. (Eds.), IX Congresso da Geografia Portuguesa- Geografia: Espaço, Natureza, Sociedade e Ciência, p.822-827, Evora, Portugal, 28-30 Novembro, 2013. ISBN:978972-99436-6-9 Disponível online no endereço url: https://sites.google. com/a/campus.ul.pt/tsurima/publications (acedido em 1 Fevereiro, 2014) 
SCHMIDTLEIN, Mathew; et al. (2008) - "A Sensitivity Analysis of the Social Vulnerability Index". Risk Analysis 28(4) 1099-1114. Disponível online no endereço url: http://onlinelibrary.wiley.com/doi/10.1111/j.15396924.2008.01072.x/full (acedido em 1 Fevereiro, 2014)

TAVARES, Alexandre Oliveira et al. (2011) - "Percepção dos riscos naturais e tecnológicos, confiança institucional e preparação para situações de emergência: O caso de Portugal continental”. Revista Crítica de Ciências Sociais, 93. 167-193 Disponível online no endereço url: http://rccs.revues.org/1380 (acedido em 1 Fevereiro, 2014) 
Série Documentos

Imprensa da Universidade de Coimbra

Coimbra University Press

2015

- U M

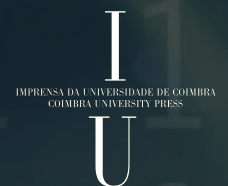

